12. Broers, J.L., Hutchison, C.J., and Ramaekers, F.C. 2004. Laminopathies. J. Pathol. 204:478-488.

13. Fidzianska, A., and Hausmanowa-Petrusewicz, I. 2003. Architectural abnormalities in muscle nuclei. Ultrastructural differences between X-linked and autosomal dominant forms of EDMD. J. Neurol. Sci. 210:47-51.

14. Fidzianska, A., Toniolo, D., and HausmanowaPetrusewicz, I. 1998. Ultrastructural abnormality of sarcolemmal nuclei in Emery-Dreifuss muscular dystrophy (EDMD). J. Neurol. Sci. 159:88-93.

15. Ostlund, C., and Worman, H.J. 2003. Nuclear envelope proteins and neuromuscular diseases. Muscle Nerve. 27:393-406

16. Sullivan, T., et al. 1999. Loss of A-type lamin expression compromises nuclear envelope integrity leading to muscular dystrophy. J. Cell Biol. 147:913-920.

17. Sylvius, N., et al. 2005. In vivo and in vitro examination of the functional significances of novel lamin gene mutations in heart failure patients. J. Med. Genet. 42:639-647.
18. Broers, J.L., et al. 2004. Decreased mechanical stiffness in LMNA-/- cells is caused by defective nucleo-cytoskeletal integrity: implications for the development of laminopathies. Hum. Mol. Genet. 13:2567-2580

19. Lammerding, J., et al. 2006. Lamins A and C but not lamin B1 regulate nuclear mechanics. J. Biol. Chem. 281:25768-25780.

20. Lammerding, J., et al. 2005. Abnormal nuclear shape and impaired mechanotransduction in emerin-deficient cells. J. Cell Biol. 170:781-791.

21. Lammerding, J., et al. 2004. Lamin A/C deficiency causes defective nuclear mechanics and mechanotransduction. J. Clin. Invest. 113:370-378. doi:10.1172/JCI200419670.

22. Heydemann, A., Demonbreun, A., Hadhazy, M., Earley, J.U., and McNally, E.M. 2007. Nuclear sequestration of delta-sarcoglycan disrupts the nuclear localization of lamin $\mathrm{A} / \mathrm{C}$ and emerin in cardiomyocytes. Hum. Mol. Genet. 16:355-363.

23. Yasuda, S., et al. 2005. Dystrophic heart failure blocked by membrane sealant poloxamer. Nature. 436:1025-1029.

24. Quinlan, J.G., et al. 2006. Poloxamer 188 failed to prevent exercise-induced membrane breakdown in mdx skeletal muscle fibers. Neuromuscul. Disord. 16:855-864.

25. Jewell, R.C., Khor, S.P., Kisor, D.F., LaCroix, K.A. and Wargin, W.A. 1997. Pharmacokinetics of RheothRx injection in healthy male volunteers. J. Pharm. Sci. 86:808-812.

26. Davis, D.B., Delmonte, A.J., Ly, C.T., and McNally, E.M. 2000. Myoferlin, a candidate gene and potential modifier of muscular dystrophy. Hum. Mol. Genet. 9:217-226.

27. Bansal, D., and Campbell, K.P. 2004. Dysferlin and the plasma membrane repair in muscular dystrophy. Trends Cell Biol. 14:206-213.

28. Dalakas, M.C., et al. 2000. Desmin myopathy, a skeletal myopathy with cardiomyopathy caused by mutations in the desmin gene. $N$. Engl. J. Med. 342:770-780.

\title{
p53, chemokines, and squamous cell carcinoma
}

\author{
David M. Owens
}

Departments of Dermatology and Pathology, Columbia University College of Physicians and Surgeons, New York, New York, USA.

\begin{abstract}
The genetic and epigenetic events underlying cutaneous squamous cell carcinoma (SCC) have been actively studied; however, no resulting preventative or therapeutic strategies have successfully targeted this lesion, apart from surgery. In this issue of the JCI, two novel regulators of SCC pathogenesis are introduced, gain-of-function mutations in the $p 53$ gene, reported by Caulin et al., and chemokine sequestration by the D6 receptor, reported by Nibbs et al. (see the related articles beginning on pages 1884 and 1893, respectively). These studies provide new twists and insights into the development of this potentially lethal disease.
\end{abstract}

Nonmelanoma skin cancer (NMSC) is the most common type of human malignancy, with over one million new cases in the United States annually (1-3). NMSC includes basal cell carcinoma and squamous cell carcinoma (SCC), with SCCs constituting approximately $20 \%$ of all NMSCs. In contrast to basal cell carcinomas, SCCs characteristically exhibit a high propensity for invasion and metastasis and may be lethal $(1,2)$. While the genetic and epigenetic events associated with SCC pathogenesis have been extensively studied, no resulting therapeutic approaches have been generated for the prevention or treatment of this potentially lethal disease. This, coupled with the escalating incidence of NMSC over the

Nonstandard abbreviations used: NMSC, nonmelanoma skin cancer; SCC, squamous cell carcinoma.

Conflict of interest: The author has declared that no conflict of interest exists.

Citation for this article: J. Clin. Invest. 117:1752-1755 (2007). doi:10.1172/JCI32719. last few decades, has made this disease a major public health issue.

\section{The $p 53$ oncogene: implications for cutaneous SCC}

It is widely accepted that genetic insults are indispensable in the formation of a frank tumor. In the development of human cutaneous SCCs, alterations in ras genes $(10 \%-30 \%$ incidence) $(3,4)$ and the $p 53$ tumor suppressor gene $(40 \%-50 \%$ incidence) $(5,6)$ have been most heavily implicated. While the majority of these lesions are missense mutations, the functional assessment of p53 missense mutations is complicated in that some give rise to a loss-of-function or null phenotype classically associated with tumor suppressor genes, whereas the majority of $p 53$ missense mutations appear to result in a gain-of-function phenotype. While the presence of both types of p53 mutations in SCCs denotes a selection advantage to these genetic lesions, whether gain-offunction or loss-of-function mutations are more critical for SCC development is unclear. Therefore, the understanding of p53 phenotype status, i.e., tumor suppressive versus oncogenic, as it relates to SCC formation and progression is of paramount importance for the development of relevant therapeutic approaches that target this gene.

In this issue of the JCI, Caulin and coworkers (7) address this problem with an elegant transgenic mouse skin model that delivers allelic doses of oncogenic $K$-ras (8) in combination with either $p 53$ gain-of-function or null mutations under the regulatory control of an inducible Cre recombinase and targeted to the proliferative layer of the epidermis by the cytokeratin K5 promoter. Using this approach, the authors clearly show that certain p53 missense mutations occurring in human SCCs demonstrate gainof-function properties in vivo and that p53 gain-of-function mutations accelerate both the frequency and progression of SCCs genetically initiated with oncogenic ras. For over a decade, the conventional regulation of cutaneous SCC by p53 tumor suppressor function was based on the observation that reductions in p53 typically do not increase the frequency or incidence of tumor formation but rather increase the frequency of malignant conversion to SCC (9). The current study confirms this traditional role for loss-of-function p53 mutations 


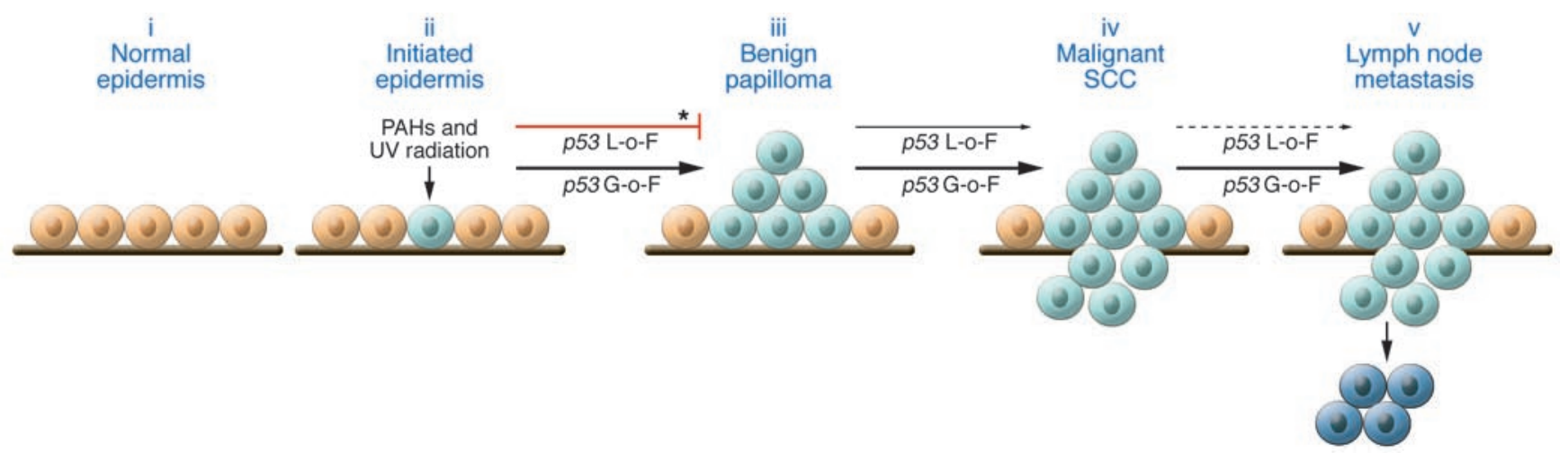

Figure 1

Impact of $p 53$ gain-of-function versus null mutations in epidermal carcinogenesis. In this issue of the $\mathrm{JCl}$, Caulin et al. (7) compare the impact of $p 53$ gain-of-function (G-o-F) versus loss-of-function (L-o-F) mutations in multistage skin carcinogenesis progressing from normal epidermis (i) to initiated epidermis (ii), benign papillomas (iii), malignant SCC (iv), and finally lymph node metastasis (v). p53 gain-of-function mutations effectively stimulate early and late stages of epidermal carcinogenesis, whereas null mutations primarily influence malignant conversion to SCC. Light blue cells, initiated cells containing ras and/or p53 mutations; dark blue cells, metastatic cells; thin arrow, inducer; bold arrow, strong inducer; dotted arrow, little to no effect; asterisk, data previously demonstrated by Kemp et al. (9). PAH, polycyclic aromatic hydrocarbon.

in malignant conversion to SCC and also provides completely novel roles for gain-of-function mutant p53 in the early stages of benign papilloma formation and during late-stage metastasis (Figure 1). What is most striking about this study is the accelerated malignant progression, genomic instability, and incidence of metastasis in cells harboring p53 gain-of-function mutations versus $p 53$-null cells. These results indicate that p53 has potent oncogenic properties, and in addition to the p53 mutation spectra that result in the null phenotype critical for conversion to SCC, Caulin et al. have now demonstrated that $p 53$ gain-of-function mutations may actually shift the epigenetic environment of an SCC cell toward that of a highly malignant spindle cell phenotype. Spindle cell tumors, while rarely observed in mouse skin carcinogenesis models, represent the most advanced stage of SCC, are composed almost entirely of undifferen-

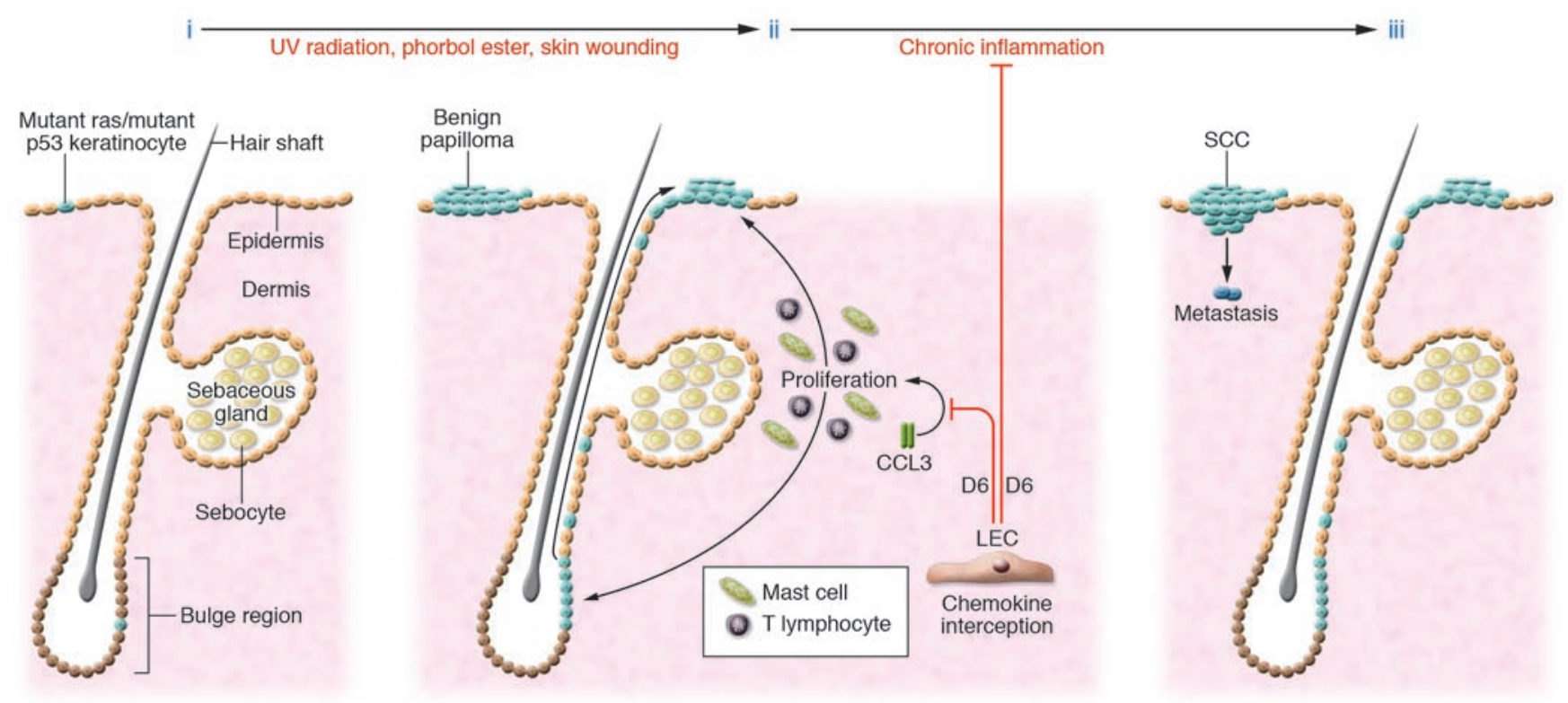

Figure 2

D6-mediated chemokine sequestration as a threshold barrier for cutaneous SCC. (i) Basal cells in the interfollicular epidermis and hair follicles sustain mutations (light blue cells) due to exposure to UV radiation, chemical carcinogens, viruses, and other pathogens; however, without epigenetic intervention, most of these mutations will lay dormant. (ii) Repeated skin irritation or wounding can heighten inflammatory chemokine levels over that which can be sustained through interception by the D6 receptor (expressed by lymphatic endothelial cells [LECs] in the dermis), resulting in the exacerbated levels of mast cells and T lymphocytes critical for stimulating benign papilloma formation. (iii) In the chronic situation, high levels of immune cells are maintained in the skin and enhance malignant conversion and metastasis of neoplastic epidermal cells (dark blue). 
tiated cells, and characteristically exhibit a highly aggressive and invasive behavior. Since p53 gain-of-function mutants are transcriptionally active in vitro (10), further studies are needed to identify the potential therapeutic targets within the unique mRNA pool that is maintained by these gain-of-function mutants in vivo. As noted by the authors, a relatively high number of cells harboring p53 missense mutations exist in sun-exposed human epidermis but ultimately never result in SCC formation (6). This supports the multi-hit carcinogenesis model and the hypothesis that additional genetic alterations are required for the development of SCC. However, future studies that allow for more precise targeting of $p 53$ gainof-function mutations in the epidermis may also illustrate the importance of target cell phenotype, i.e., stem cell versus progenitor or transit amplifying cell, in p53-driven SCC formation.

\section{D6: an atypical chemokine receptor}

In general, the relationship between inflammation and cancer has been well documented, from the influence of chronic inflammatory agents on neoplastic cell proliferation in founding a tumor to later stages of tumor progression associated with immune suppression, and clearly indicates that both innate and adaptive responses of the immune system are deeply rooted in cancer cell growth, progression, and migration (11).

A link between skin inflammation and SCC formation was reported as early as 1828 as Marjolin ulcer (12), where SCC development was observed at sites of chronic skin inflammation induced by thermal injury. More recently, the association between SCC development and inflammation has been extended to several human skin pathologies associated with ulceration and further substantiated in a number of transgenic murine skin carcinogenesis models (see ref. 13 for an excellent review), collectively indicating that chronic inflammatory stimuli may be a driving force for epidermal neoplastic cell progression. Despite these efforts, very few studies have surfaced that identify specific inflammatory molecules that are required for de novo skin carcinogenesis (14-16).

In this issue of the JCI, Nibbs and coworkers (17) have assessed the requirement of the atypical chemokine receptor D6 in chemically induced skin inflamma- tion and SCC formation. Previously, this group and others have shown that D6 regulates skin inflammatory responses by reducing the bioavailability of inflammatory chemokines (18), and in the present study they postulate that the sequestration of inflammatory chemokines by D6 is essential to suppressing epidermal carcinogenesis. To support their hypothesis, they show that D6-deficient mice are acutely sensitive to chemically induced SCC formation and in doing so provide the first in vivo evidence that chemokine sequestration is essential for suppressing cutaneous SCC (17). The phenotypic response of D6-deficient mice to acute treatment with the irritant phorbol ester is consistent with the reported role for this receptor in the regulation of heightened inflammatory chemokine levels (18). Their findings in experimental mouse skin carcinogenesis were corroborated by the induction of D6 in lymphatic endothelial cells in close proximity to invasive SCC cells from human oral SCCs. In their model, production of the proinflammatory CC chemokine ligand 3 (CCL3) is prolonged in D6-deficient skin after treatment with phorbol ester, leading to chronic recruitment of two cell types known to be involved in cutaneous SCCs, $\mathrm{CD}^{+} \mathrm{T}$ cells and dermal mast cells (Figure 2). In the absence of D6 chemokine sequestration, the exacerbation of the proinflammatory epidermal microenvironment sensitizes the epidermis to hyperproliferation and ras-driven tumor formation (Figure 2). Finally, the persistence of the proinflammatory chemokine response maintains chronic recruitment of lymphocytes and mast cells and stimulates SCC invasion and metastasis (Figure 2). According to this model, the ability of a particular inflammatory agent to overcome the threshold of normal D6-mediated CCL3 interception should directly correlate with tumor-promoting capability.

\section{Are p53 and inflammatory chemokines linked?}

Collectively, these studies $(7,17)$ give critical insight into two apparently distinct therapeutic approaches to potentially target cutaneous SCC. However, the induction of late-stage metastases common to both p53 and D6 transgenic mouse models is normally a rare event in experimental mouse skin carcinogenesis and therefore raises the question as to whether there is a more direct relation- ship between certain proinflammatory signals and p53 gain-of-function mutations as they relate to SCC pathogenesis. For example, can a heightened inflammatory chemokine response critically influence the $p 53$ status in otherwise normal epidermal cells and/or pre-neoplastic epidermal cells that have sustained additional genetic insults? This concept fits well with the UV carcinogenesis model in human skin, in which small clusters of p53 mutant cells exist prior to actinic keratosis (AK) and SCC development (6) and may be selected for clonal expansion by a chronic inflammatory stimulus (13). In addition, $\mathrm{AK}$ are reported to undergo a short inflammatory phase just prior to conversion to SCC that is accompanied by an increase in p53 levels (19). If a heightened inflammatory state in the skin can influence $p 53$ gain-of-function status, this may have important implications for the prognosis of cutaneous SCCs associated with skin inflammatory pathologies. Although the precursor to chemically induced murine SCCs, squamous papilloma, does not typically harbor $p 53$ mutations, increased levels of wild-type p53 are observed (20). A better understanding of the impact of D6mediated chemokine sequestration on UV-induced carcinogenesis may help to address this concept.

Address correspondence to: David M. Owens, Department of Dermatology, Columbia University College of Physicians and Surgeons, 630 West 168th Street, VC15-202, New York, New York 10032, USA. Phone: (212) 851-4544; Fax: (212) 851-4540; E-mail: do2112@columbia.edu.

1. Murad, A., and Ratner, D. 2001. Primary care: cutaneous squamous-cell carcinoma. N. Engl. J. Med. 344:975-983.

2. Albert, M.R., and Weinstock, M.A. 2003. Keratinocyte carcinoma. CA Cancer J. Clin. 53:292-302.

3. Pierceall, W.E., Goldberg, L.H., Tainsky, M.A., Mukhopadhyay, T., and Ananthaswamy, H.N. 1991. Ras gene mutation and amplification in human nonmelanoma skin cancers. Mol. Carcinog. 4:196-202.

4. Spencer, J.M., Kahn, S.M., Jiang, W., DeLeo, V.A., and Weinstein, I.B. 1995. Activated ras genes occur in human actinic keratoses, premalignant precursors to squamous cell carcinomas. Arch. Dermatol. 131:796-800.

5. Bolshakov, S., et al. 2003. p53 mutations in human aggressive and nonaggresive basal and squamous cell carcinomas. Clin. Cancer Res. 9:228-234.

6. Brash, D.E., et al. 1991. A role for sunlight in skin cancer: UV-induced p53 mutations in squamous cell carcinoma. Proc. Natl. Acad. Sci. U. S. A. 88:10124-10128

7. Caulin, C., et al. 2007. An inducible mouse model for skin cancer reveals distinct roles for gain- and loss-of-function p53 mutations. J. Clin. Invest. 
117:1893-1901. doi:10.1172/JCI31721.

8. Jackson, E.L., et al. 2001. Analysis of lung tumor initiation and progression using conditional expression of oncogenic K-ras. Genes Dev. 15:3243-3248.

9. Kemp, C.J., Donehower, L.A., Bradley, A., and Balmain, A. 1993. Reduction of p53 gene dosage does not increase initiation or promotion but enhances malignant progression of chemically induced skin tumors. Cell. 74:813-822.

10. Dittmer, D., et al. 1993. Gain of function mutations in p53. Nat. Genet. 4:42-46.

11. Coussens, L.M., and Werb, Z. 2002. Inflammation and cancer. Nature. 420:860-867.

12. Marjolin, J.N. 1828. Ulcère [In French]. In Dictionnaire de médecine. Paris Béchet jeune. Paris, France. 31-50.

13. Mueller, M.M. 2006. Inflammation in epithelial skin tumours: old stories and new ideas. Eur. J. Cancer. 42:735-744.

14. Moore, R.J., et al. 1999. Mice deficient in tumor necrosis factor-alpha are resistant to skin carcinogenesis. Nat. Med. 5:828-831.

15. Coussens, L.M., Tinkle, C.L., Hanahan, D., and Werb, Z. 2000. MMP-9 supplied by bone marrowderived cells contributes to skin carcinogenesis. Cell. 103:481-490.

16. Girardi, M., et al. 2001. Regulation of cutaneous malignancy by gammadelta $\mathrm{T}$ cells. Science. 294:605-609.
17. Nibbs, R.J.B., et al. 2007. The atypical chemokine receptor D6 suppresses the development of chemically induced skin tumors. J. Clin. Invest. 117:1884-1892. doi:10.1172/JCI30068.

18. Jamieson, T., et al. 2005. The chemokine receptor D6 limits the inflammatory response in vivo. Nat. Immunol. 6:403-411.

19. Berhane, T., Halliday, G.M., Cooke, B., and Barnetson, R.S. 2002. Inflammation is associated with progression of actinic keratoses to squamous cell carcinomas in humans. Br. J. Dermatol. 146:810-815.

20. Owens, D.M., Wei, S.-J.C., and Smart, R.C. 1999. A multihit, multistage model of chemical carcinogenesis. Carcinogenesis. 20:1837-1844.

\title{
Hepcidin regulation: ironing out the details
}

\author{
Ivana De Domenico, Diane M. Ward, and Jerry Kaplan
}

Department of Pathology, University of Utah School of Medicine, Salt Lake City, Utah, USA.

\begin{abstract}
Hepcidin is a peptide hormone secreted by the liver that plays a central role in the regulation of iron homeostasis. Increased hepcidin levels result in anemia while decreased expression is the causative feature in most primary iron overload diseases. Mutations in hemochromatosis type 2 (HFE2), which encodes the protein hemojuvelin (HJV), result in the absence of hepcidin and an early-onset form of iron overload disease. HJV is a bone morphogenetic protein (BMP) coreceptor and HJV mutants have impaired BMP signaling. In this issue of the JCI, Babitt and colleagues show that BMPs are autocrine hormones that induce hepcidin expression (see the related article beginning on page 1933). Administration of a recombinant, soluble form of $\mathrm{HJV}$ decreased hepcidin expression and increased serum iron levels by mobilizing iron from splenic stores. These results demonstrate that recombinant $\mathrm{HJV}$ may be a useful therapeutic agent for treatment of the anemia of chronic disease, a disorder resulting from high levels of hepcidin expression.
\end{abstract}

\section{Regulation of iron homeostasis}

In all species, the concentration of iron in biological fluids is tightly regulated to provide iron as needed and to avoid toxicity, because iron excess can lead to the generation of reactive oxygen species. Iron homeostasis in mammals is regulated at the level of intestinal absorption, as there is no excretory pathway for iron. Hepcidin, a circulating peptide hormone, is the master regulator of systemic iron homeostasis, coordinating the use and storage of iron with iron acquisition (1). This hormone is primarily produced by hepatocytes and is a negative regulator of iron entry into plasma (Figure 1). Hepcidin acts by binding to ferroportin, an iron

Nonstandard abbreviations used: BMP, bone morphogenetic protein; HJV, hemojuvelin.

Conflict of interest: The authors have declared that no conflict of interest exists.

Citation for this article: J. Clin. Invest. 117:1755-1758 (2007). doi:10.1172/JCI32701. transporter present on cells of the intestinal duodenum, macrophages, and cells of the placenta. Binding of hepcidin induces ferroportin internalization and degradation (2). The loss of ferroportin from the cell surface prevents iron entry into plasma (Figure 1A). Decreased iron entry into plasma results in low transferrin saturation, and less iron is delivered to the developing erythroblast. Conversely, decreased expression of hepcidin leads to increased cell surface ferroportin and increased iron absorption (Figure 1C).

Plasma hepcidin levels are regulated by different stimuli, including cytokines, plasma iron, anemia, and hypoxia. Dysregulation of hepcidin expression results in iron disorders. Overexpression of hepcidin leads to the anemia of chronic disease, while low hepcidin production results in hereditary hemochromatosis with consequent iron accumulation in vital organs (Figure 1). Most hereditary iron disorders result from inadequate hepcidin pro- duction relative to the degree of tissue iron accumulation. Impaired hepcidin expression has been shown to result from mutations in any of 4 different genes: transferrin receptor 2 (TFR2), hemochromatosis (HFE), hemochromatosis type 2 (HFE2), and hepcidin antimicrobial peptide (HAMP). Mutations in HAMP, the gene that encodes hepcidin, result in iron overload disease, as the absence of hepcidin permits constitutively high iron absorption. The role for other genes (TFR2, HFE, and HFE2) in the regulation of hepcidin production has been unclear. A study presented in this issue of the JCI by Babitt et al. (3) provides a mechanistic understanding of the role of hemojuvelin (HJV; encoded by HFE2) in the regulation of hepcidin production.

\section{Regulation of hepcidin expression}

Regulation of hepcidin expression appears to occur at the level of transcription (Figure 2). Inflammatory cytokines, predominately IL-6, induce transcription of HAMP in hepatocytes. This induction involves the activation of Stat3 (3) and binding of Stat 3 to a regulatory element in the HAMP promoter (4-6).

A second mode of hepcidin regulation depends upon signaling through the bone morphogenetic protein/Smad (BMP/Smad) pathway. BMPs are cytokines in the TGF- $\beta$ family. These ligands play key roles in regulating cell proliferation, differentiation, apoptosis, and migration in tissues. In particular, BMPs play essential roles in cardiac, neural, and cartilaginous differentiation. All BMPs share a common signaling pathway, which involves 